\title{
Osteoporosis and Endplate Damage Correlation Using a Combined Approach of Hounsfield Unit Values and Total Endplate Scores: A Retrospective Cross-Sectional Study
}

\author{
Chenyang Zhuangl,* \\ Zixiang Wang',* \\ Weisin Chen' \\ Bo Tian' \\ Juan $\mathrm{Li}^{1,2}$ \\ Hong Lin ${ }^{1,2}$ \\ 'Department of Orthopedics, Zhongshan \\ Hospital, Fudan University, Shanghai, \\ People's Republic of China; ${ }^{2}$ Department \\ of Orthopedics, Shanghai Geriatrics \\ Center, Fudan University, Shanghai, \\ People's Republic of China \\ *These authors contributed equally to \\ this work
}

Correspondence: Juan $\mathrm{Li}$

Department of Orthopedics, Zhongshan

Hospital, Fudan University, No. 180

Fenglin Road, Shanghai, 200032, People's

Republic of China

$\mathrm{Tel} / \mathrm{Fax}+862164041990$

Email li_juan1983@outlook.com

Hong Lin

Department of Orthopedics, Shanghai

Geriatrics Center, Fudan University,

No. 2560 Chunshen Road, Shanghai,

201 104, People's Republic of China

Email lin.hongl@zs-hospital.sh.cn

\begin{abstract}
Purpose: Osteoporosis and endplate damage, two primary orthopedic disorders that have adverse effects on the quality of life of older adults, may have some previously unknown relationship. The purpose of this study was to determine the potential association between osteoporosis and endplate damage with two specific imaging scoring systems and analyze the underlying mechanisms.

Patients and Methods: A cross-sectional study including 156 patients with degenerative disc disease (DDD) who visited our department in 2018 was performed. Data including age, sex, body mass index, Hounsfield unit (HU) values utilizing computed tomography (CT), and total endplate scores (TEPSs) using magnetic resonance imaging (MRI) of all patients were retrospectively collected and analyzed. The average HU value and TEPS of L1-L4 were used to represent the degrees of bone mineral density (BMD) and endplate damage, respectively. Patients with an $\mathrm{HU}$ value $<110$ were defined as having osteoporosis and placed in the lowBMD group; otherwise, they were placed in the normal-BMD group. Multivariate logistic regression models were used to determine the independent factors of endplate damage.

Results: The TEPSs in the low-BMD group were significantly higher $(6.4 \pm 1.6$ vs $5.0 \pm 0.9$, $\mathrm{p}<0.001)$ overall and in every segment of L1-L4 $(\mathrm{p}<0.01)$. A significant negative correlation was found between TEPS and HU values $(\mathrm{p}<0.001)$. The HU value (odds ratio [OR] 0.221; 95\% confidence interval [CI], 0.148-0.295, $\mathrm{p}<0.001$ ), age (OR 0.047; 95\% CI, 0.029-0.224, p < 0.001), and BMD (OR 3.796; 95\% CI, 2.11-7.382, $<<0.05$ ) were independent factors influencing endplate damage.

Conclusion: A significantly positive correlation was observed between osteoporosis and endplate damage, indicating the requirement for a more comprehensive therapeutic regimen for treating patients with DDD complicated with osteoporosis.
\end{abstract}

Keywords: degenerative disc diseases, bone mineral density, computed tomography, magnetic resonance imaging

\section{Introduction}

Osteoporosis and degenerative disc diseases (DDD) are common aging-related diseases in the world. In China, the prevalence of osteoporosis in people aged over 50 years is $20 \%$ in women and $14 \%$ in men. ${ }^{1}$ It was also estimated that approximately 12.3 million people older than 50 years in the US would face the threat of osteoporosis by $2020 .{ }^{2}$ Meanwhile, the incidence of DDD has also increased in the recent years, affecting $50-90 \%$ of people over 70 years of age, according to several reports. ${ }^{3-5}$ 
The vertebral endplate is a thin bilayer between the vertebrae and the intervertebral disc, playing an important role in the nutrition supply and stress conduction of the intervertebral disc. ${ }^{6}$ Damage to endplates is a typical manifestation of DDD in addition to direct disc abnormality. ${ }^{7}$ Osteoporosis and endplate damage are proven risk factors for long-term low back pain, vertebral fractures, and some postoperative complications, which may induce a higher rate of disability and mortality among older adults. ${ }^{8-10}$ However, only a few studies have been conducted to determine the relationship between these conditions. Based on animal model studies, some researchers hypothesized that osteoporosis may cause further deterioration of endplates. ${ }^{11}$ Although there was a lack of appropriate methods for defining osteoporosis and endplate damage in patients with DDD, their relationship remains elusive. Hence, the exact association between osteoporosis and endplate damage is worth investigating in depth.

Dual-energy X-ray absorptiometry (DXA) is the most widely used method for screening and diagnosing osteoporosis, and the measurement of the bone mineral density (BMD) of axial bones employing DXA, with the output as the T-score, is recommended. ${ }^{12}$ However, conditions such as vertebral hyperplasia, small joint sclerosis, scoliosis, osteophytes, and pannus formation can cause mismeasurement with DXA in patients with DDD, as confirmed by many studies. ${ }^{13,14}$ Recently, an alternative method to assess the BMD of patients with DDD using Hounsfield unit (HU) values by computed tomography (CT) was introduced and recommended by many experts. ${ }^{15,16}$ Zou et al found that $\mathrm{CT}$ HU values could be used as a complementary method to identify undiagnosed spinal osteoporosis, which was easily underestimated in patients with lumbar degenerative diseases. ${ }^{16}$ Previously, Rajasekaran et al classified endplates into six types (types 1-6) according to the severity of their damage, assessed on T1-weighted magnetic resonance imaging (MRI) scans, which revealed a strong correlation with DDD. ${ }^{17} \mathrm{~A}$ total endplate score (TEPS), obtained by summing the cranial and caudal endplate scores of a single disc, was recommended to evaluate endplate damage, which plays a crucial role in precipitating DDD.

The purpose of this study was to determine the relationship between osteoporosis and endplate damage using the scoring systems mentioned above and to investigate the evidence for treating DDD accompanied with osteoporosis more comprehensively and effectively.

\section{Materials and Methods}

\section{Study Design}

This study was a retrospective cross-sectional analysis based on radiological data from a single center.

\section{Patient Selection}

PASS version 15.0 (NCSS, Kaysville, UT, USA) was used to calculate sample size, which showed a required sample size of more than 29 samples in each group. An analysis of all hospitalized patients in the Department of Orthopedics in our hospital from January 2018 to December 2018 was performed. The inclusion criteria were patients aged between 45 and 85 years; diagnosis of DDD including degenerative lumbar spinal stenosis, lumbar disc herniation, and degenerative lumbar spondylolisthesis; and patients who had undergone both lumbar MRI and three-dimensional CT within a month in our hospital before surgery. The exclusion criteria were a history of spinal surgery; the use of antiosteoporotic drugs, glucocorticoids or other medications that affect bone metabolism; those who had vertebral fractures, tumors, tuberculosis, or other infectious diseases. The indications for surgery of DDD are moderate and severe nerve compression symptoms (with or without low back pain); progressive walking restriction; progressive loss of neurological function; symptoms of cauda equina, and failed conservative treatment for 4-6 weeks. ${ }^{18}$ Demographic data such as sex, age, weight, and body mass index (BMI) were recorded. We confirmed that all involved patients gave their written and verbal informed consent prior to study inclusion, which was approved by the institutional ethical committee and all the data was anonymized and confidential. This study's protocol was approved by the institutional ethical committee of Zhongshan Hospital (B2021-388) and was performed according to the principles of the Declaration of Helsinki of 1975.

\section{Assessment of Lumbar BMD}

All patients underwent three-dimensional lumbar $\mathrm{CT}$ at our hospital (320-row computed tomography, Aquilion One, tube voltage $120 \mathrm{kV}$; Canon Medical Systems, Japan), and images of the lumbar spine were reviewed using the picture archiving and communication system (PACS). The upper, middle, and lower axial planes of each vertebral body were selected and placed in an oval region of interest (ROI) by two authors (CZ and ZW). The ROI was selected such that it included as much trabecular bone as possible and excluded cortical bone, the posterior venous plexus, or bone islands (Figure 1). The 

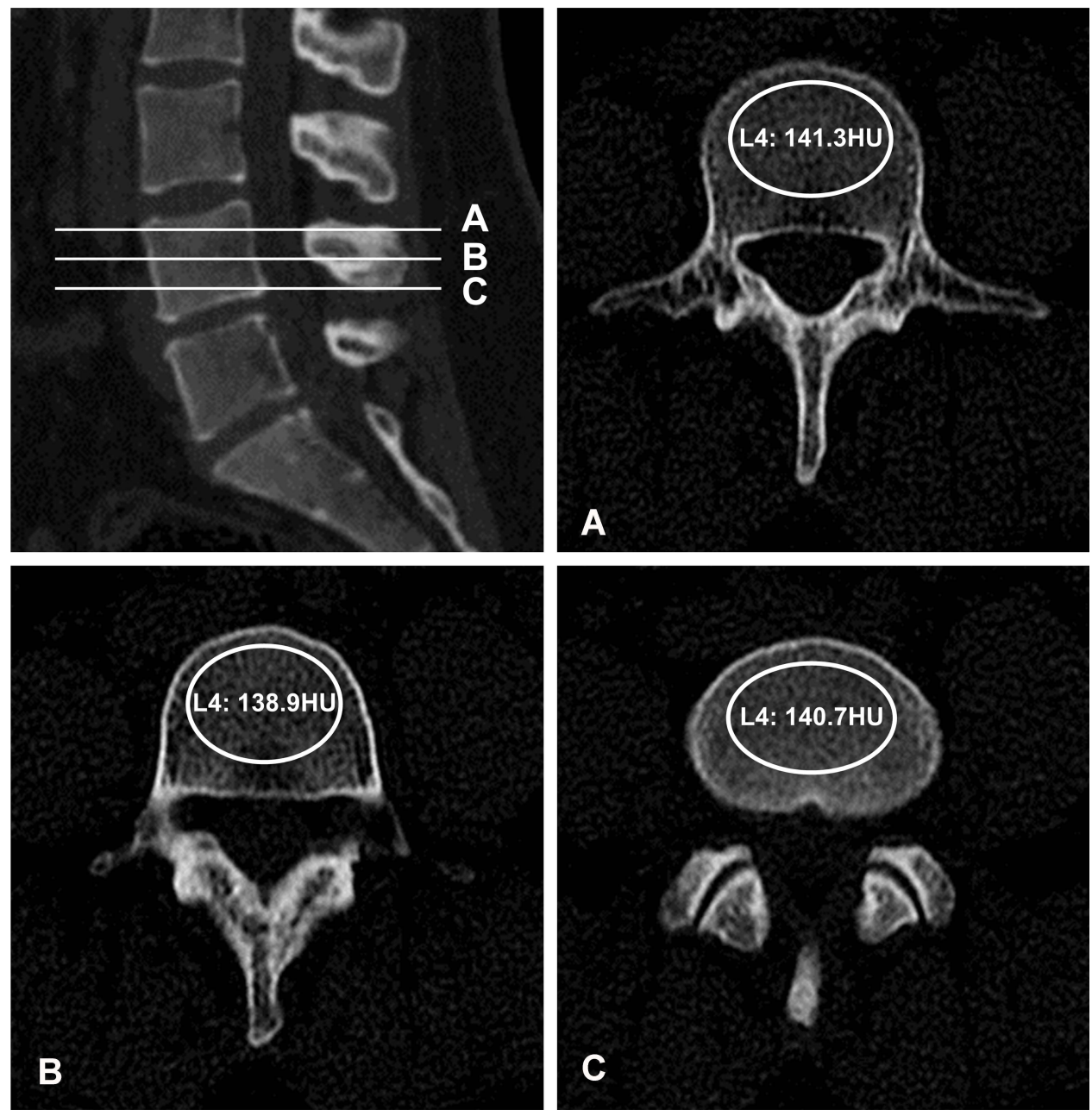

Figure I Example of measurement of Hounsfield unit $(\mathrm{HU})$ values by computed tomography. An oval region of interest is placed over the (A) upper, $(\mathbf{B})$ middle, and $(\mathbf{C})$ lower axial planes of the vertebral body, and the $\mathrm{HU}$ value is automatically calculated by picture archiving and communication system.

average HU value of L1-L4 was automatically calculated by PACS and was used to represent the bone mineral density. The threshold HU value for osteoporosis with high sensitivity and specificity was determined to be 110 according to the optimum cut-off value from previous studies. ${ }^{16,19}$

\section{Grading of Endplate Damage}

MRI scans (Magnetic Resonance Imaging System, Aera, 1.5T, Siemens, Germany) were performed on every patient, and the endplates were evaluated on T1-weighted images and were classified into six types according to the method of the previous studies by two authors $(\mathrm{CZ}$ and $\mathrm{ZW}$ ) (Figure 2). The two investigators were blinded to each other and the patients' other data. A TEPS was added with both endplate scores of every disc, and an average TEPS of L1-L4 was achieved to represent the endplate damage of each patient.

\section{Statistical Analysis}

The SPSS Statistics version 21.0 package (IBM Corp., Armonk, NY, USA) was used for data analysis. The independent samples Student's $t$-test was applied for continuous variables. A chi-squared test was applied for categorical data analysis. Analysis of variance (ANOVA) and binary linear regression were used to compare the correlation between CT HU values and TEPS. Univariate and multivariate logistic regression analyses were utilized to verify the independence of outcomes, and $\mathrm{p}<0.05$ was regarded as a significant difference.

\section{Results}

The demographic characteristics of the 156 patients included in this study are summarized in Table 1. The average HU value and TEPS were 120.4 and 5.3, respectively. 

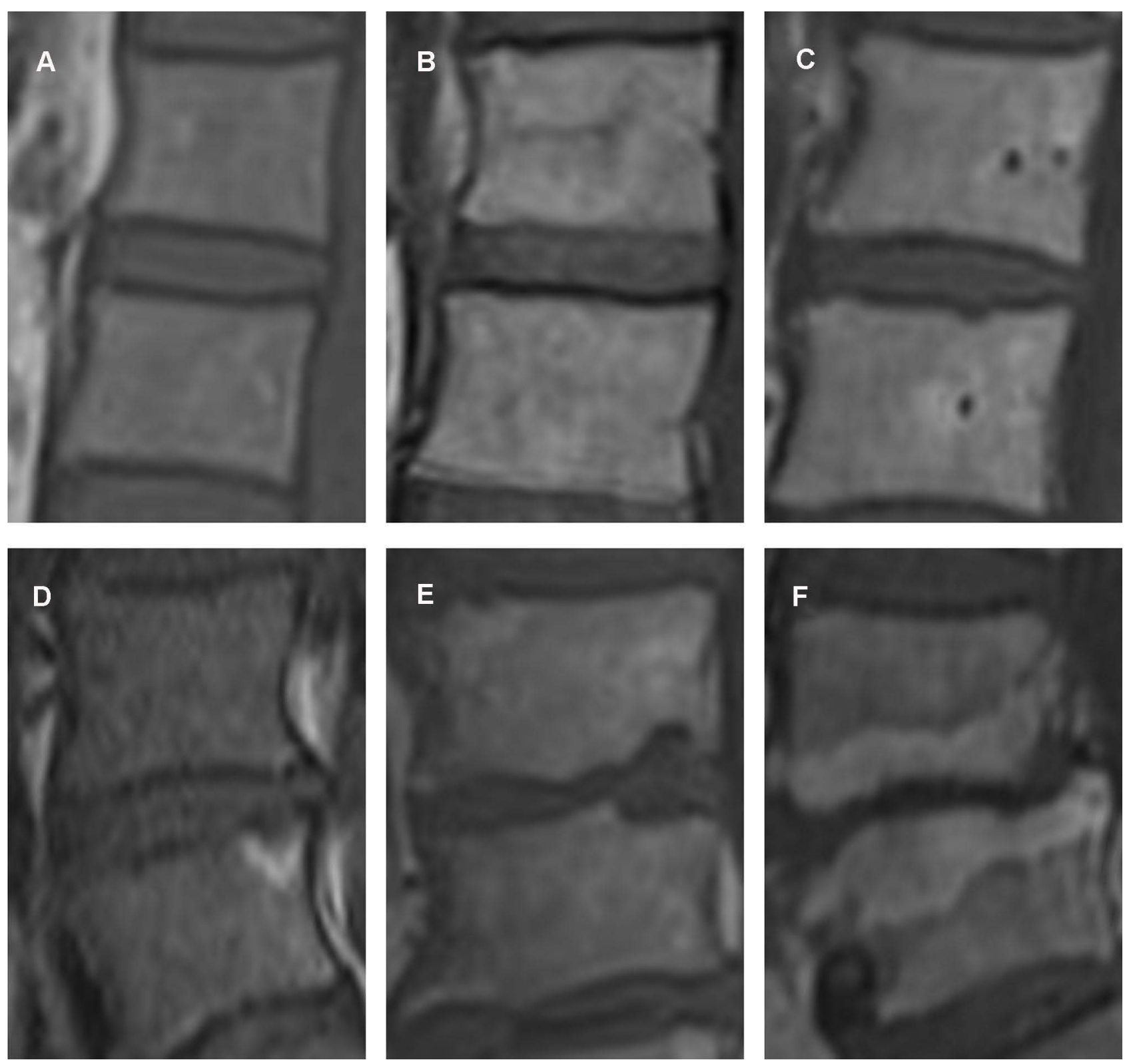

Figure 2 Example of the endplate scoring system: (A) grade I: normal endplate without breaks or defects; (B) grade 2: focal thinning without endplate breaks or defects; (C) grade 3: focal disc marrow contacts with the normal endplate contour maintained; (D) grade 4: defect or damage to up to $25 \%$ of the total endplate area; (E) grade 5: defect or damage to up to $50 \%$ of the total endplate area; (F) grade 6: complete damage of the endplate area.

The patients were divided into low- and normal-BMD groups according to their average HU values. Comparisons of TEPS and other data between the two groups are illustrated in Table 2. We found significant age differences $(68.8$ \pm 8.7 years vs $57.4 \pm 12.8$ years, $\mathrm{p}<0.001$ ), sex (Male/ Female) (29/51 vs 50/26, p <0.001), body mass index (BMI) $(24.7 \pm 3.8$ vs $25.2 \pm 2.9, \mathrm{p}<0.05)$, and TEPS $(6.4 \pm 1.6$ vs $5.0 \pm 0.9, \mathrm{p}<0.001)$ with ANOVA analysis, and except for $\mathrm{BMI}$, the remaining three indicators were independent influencing factors of low bone mineral density according to the logistic regression analysis $(\mathrm{p}<0.001)$.
We then compared the TEPSs of the two groups in every segment, and as presented in Figure 3, all TEPSs were significantly higher in the low-BMD group than in the normal-BMD group in $\mathrm{L} 1 / 2-\mathrm{L} 4 / 5(\mathrm{p}<0.01)$. The correlations between TEPS and HU values were significantly negative $(\mathrm{p}<0.001)$, as revealed by scatter plots and analyzed with linear regression (Figure 4).

To further determine the relationship between lumbar vertebral bone mineral density and endplate damage, we analyzed the correlation between TEPS and indicators including age, sex, HU value, BMI, and BMD applying univariate and 
Table I General Information

\begin{tabular}{|l|l|}
\hline Characteristics & All $(\mathbf{n}=1 \mathbf{5 6})$ \\
\hline Age(years) & $63.2 \pm 12.2(45-85)$ \\
\hline $\begin{array}{l}\text { Sex } \\
\text { Female } \\
\text { Male }\end{array}$ & 77 \\
\hline Height $(\mathrm{cm})$ & 79 \\
\hline Weight $(\mathrm{kg})$ & $164.9 \pm 7.9$ \\
\hline BMI (kg/m2) & $68.0 \pm 11.5$ \\
\hline HU value & $24.9 \pm 3.4$ \\
\hline Total endplate score & $120.4 \pm 48.7$ \\
\hline
\end{tabular}

Notes: Values are presented as mean \pm standard deviation.

Abbreviations: BMI, body mass index; HU, Hounsfield unit.

multivariate logistic regression, which are presented in Table 3. After excluding other confounding factors, HU value (OR 0.221; 95\% CI, 0.148-0.295, p < 0.001), age (OR 0.047; 95\% CI, 0.029-0.224, p < 0.001), and BMD (OR 3.796; 95\% CI, 2.11-7.382, p < 0.05) were found to be independent factors influencing endplate damage.

\section{Discussion}

Cartilaginous endplates, a type of hyaline cartilage, are thin horizontal structures that interface with the intervertebral disc and the vertebral body. Similar to the articular cartilage that also belongs to hyaline cartilage family, the cartilaginous endplate mainly comprises collagen II and aggrecan and has the characteristics of high tensile strength and elasticity. ${ }^{20}$ The endplate plays an important role in the nutrient supply and metabolite exchange of the intervertebral disc. Horner et al indicated that reduced nutrient supply and accumulation of metabolic products can lead to apoptosis of nucleus pulposus cells and promote disc degeneration. ${ }^{21}$ Rade et al also indicated that endplate damage is the initiating factor for disc degeneration. ${ }^{7}$ The relationship between osteoporosis and articular cartilage has been studied by many researchers. Calvo et al and Bertuglia et al stated that osteoporosis increased the severity of damage in the cartilage and verified the same with animal models, with similar results as those of a cross-sectional study by Çarlı et al. ${ }^{22-24}$ Although the cartilaginous endplate and the articular cartilage have many specific characteristics in common, only a few studies have reported the potential bond between endplate damage and osteoporosis. Ding et al hypothesized a significant association between osteoporosis and vertebral cartilaginous endplate lesions, verifying it with an ovariectomy rat model. ${ }^{11}$ Nevertheless, further clinical evidence in humans was required to confirm the hypothesis.

Osteoporosis is quite common among patients with lumbar fusion in lumbar degenerative diseases, and it has been indicated that the prevalence of DDD combined with osteoporosis is relatively high among older adults. $^{25}$ Moreover, as a metabolic osteopathy, osteoporosis is not exclusively prevalent in the elderly. ${ }^{26}$ Thus, timely screening and recognition of patients with DDD and osteoporosis are crucial for clinicians to select optimal treatment schemes. However, the exact relationship between these two typical abnormalities remains controversial. Many studies have focused on the correlation between osteoporosis and vertebral disc degeneration. $^{27-31}$ Surprisingly, opposing results have been reported by different studies. Salo et al and Wang et al reported that lower lumbar spine BMD was associated with less severe disc degeneration. ${ }^{29,30}$ In contrast, Harada et al and Grams et al reached a common conclusion on the inverse correlation between bone mass and vertebral disc degeneration. ${ }^{27,28}$ Furthermore, some studies have confirmed no association between these two pathological indicators. $^{31}$

One possible reason for the difference in results is methodological heterogeneity. DXA is the most common and practical tool chosen by many surgeons to evaluate and identify osteoporosis in patients. However, we could

Table 2 Comparison of Patient Data Between the Low- and Normal-BMD Groups

\begin{tabular}{|l|l|l|l|l|}
\hline Demographics & Low-BMD Group (N=80) & Normal-BMD Group (N=76) & p Value $^{\mathbf{a}}$ \\
\hline Age (years) & $68.8 \pm 8.7$ & $57.4 \pm 12.8$ & $<0.001$ \\
Sex (male/female) & $29: 51$ & $50: 26$ & $<0.001$ \\
BMI (kg/m2) & $24.7 \pm 3.8$ & $25.2 \pm 2.9$ & $<0.05$ \\
Total endplate score & $6.4 \pm 1.6$ & $5.0 \pm 0.9$ & $<0.001$ \\
\end{tabular}

Notes: anivariate analysis of variance, 'bultivariate logistic regressions.

Abbreviations: BMD, bone mineral density; BMI, body mass index. 


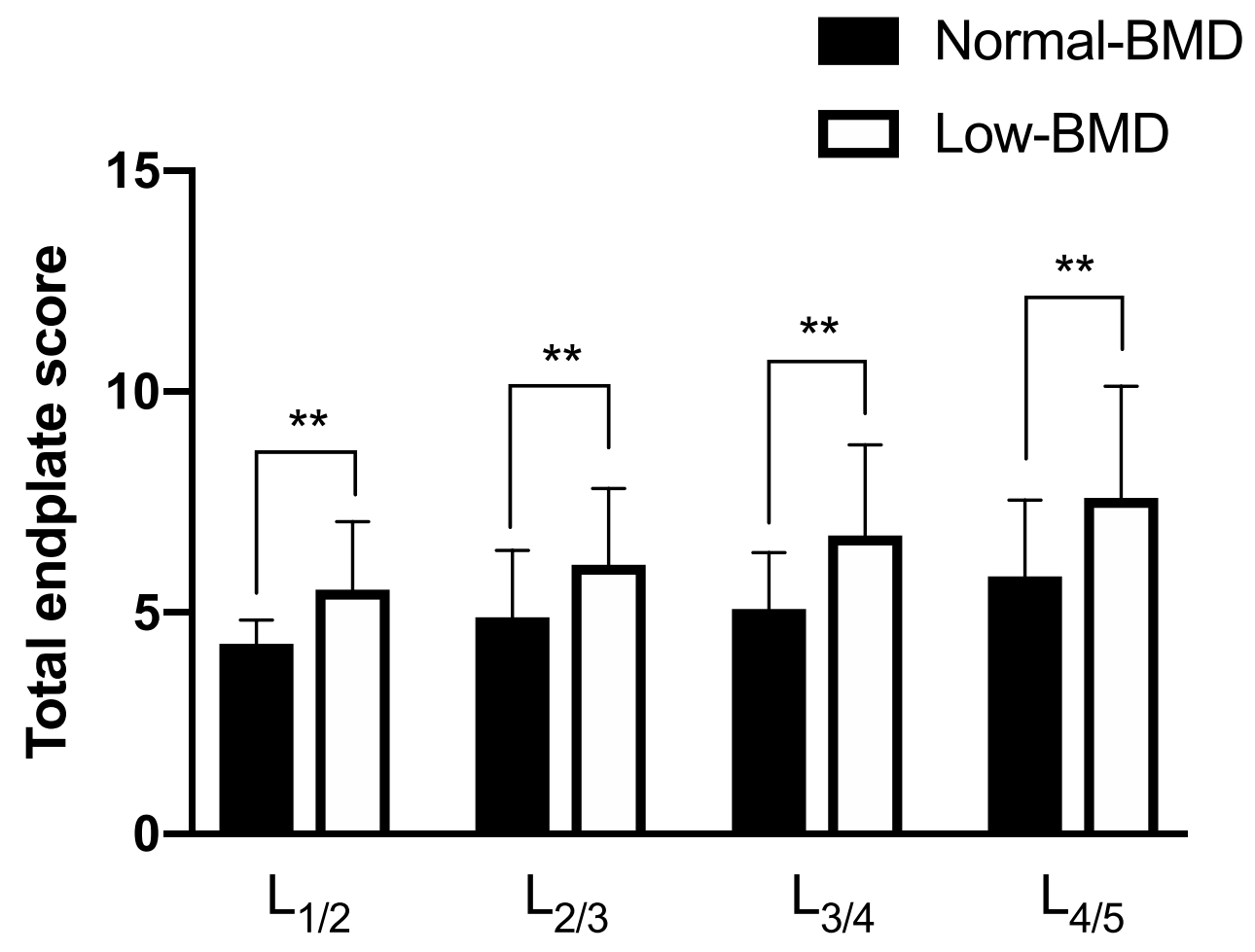

Figure 3 Comparison of the total endplate scores between the normal- and low-BMD groups in every segment in LI/2-L4/5. Note: ${ }^{* *}$ p-value $<0.01$.

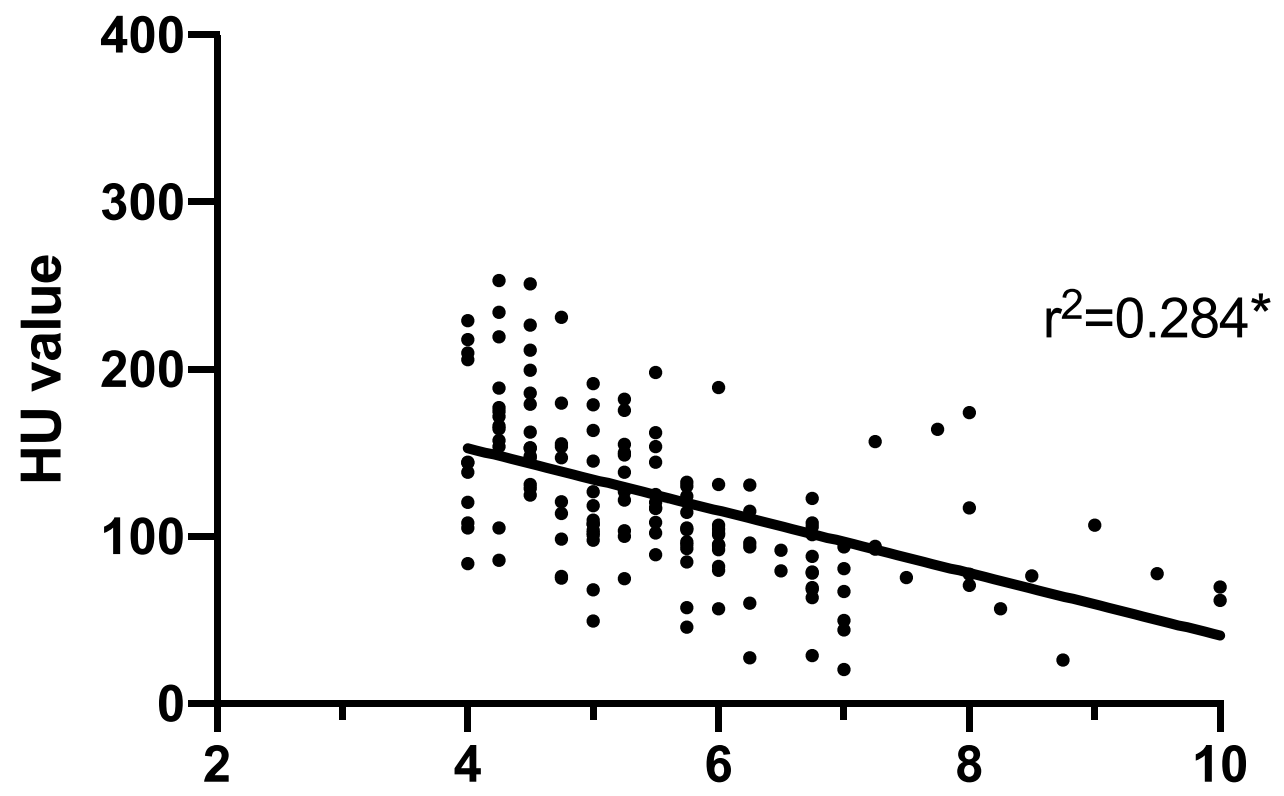

Total endplate score

Figure 4 Linear correlation (black line) between the Hounsfield unit (HU) values and total endplate scores (TEPS), and the correlation coefficient ( $\mathrm{R}^{2}$ ). Significant correlation coefficients are marked with an asterisk.

not obtain satisfactory results utilizing DXA while treating patients with degenerative diseases, which was confirmed by many previous studies. ${ }^{13,14,32,33}$ Quantitative CT (QCT) was introduced as a reliable method of assessing the bone quality with trabecular BMD instead of areal BMD measured by DXA. ${ }^{34}$ However, it cannot be performed 
Table 3 Univariate and Multivariate Associations of the Total Endplate Scores with the HU Value and Other Variables Considered

\begin{tabular}{|c|c|c|c|c|}
\hline \multirow[t]{2}{*}{ Characteristics } & \multicolumn{2}{|c|}{ Univariate Association $^{\mathrm{a}}$} & \multicolumn{2}{|c|}{ Multivariate Association ${ }^{\mathbf{b}}$} \\
\hline & OR $(95 \% \mathrm{Cl})$ & p value & OR $(95 \% \mathrm{CI})$ & $p$ value \\
\hline Age & $0.12 I(0.07 \mid-0.372)$ & $<0.001$ & $0.047(0.029-0.224)$ & $<0.001$ \\
\hline \multicolumn{5}{|l|}{ Sex } \\
\hline Male & Reference & - & Reference & - \\
\hline Female & $0.70 \mathrm{I}(0.4 \mathrm{I}-0.97)$ & $<0.001$ & $0.989(0.95-1.641)$ & 0.601 \\
\hline $\mathrm{HU}$ value & $0.622(0.510-0.923)$ & $<0.001$ & $0.221(0.148-0.295)$ & $<0.001$ \\
\hline BMI (kg/m2) & $0.221(0.102-0.491)$ & 0.112 & $0.037(0.129-0.203)$ & 0.662 \\
\hline \multicolumn{5}{|l|}{ BMD } \\
\hline Low & Reference & - & Reference & - \\
\hline Normal & $5.102(2.339-7.901)$ & 0.027 & $3.796(2.11-7.382)$ & 0.038 \\
\hline
\end{tabular}

Notes: a Univariate logistic regressions, ${ }^{\mathrm{b}}$ Multivariate logistic regressions.

Abbreviations: $\mathrm{HU}$, Hounsfield unit; OR, odds ratio; $95 \% \mathrm{Cl}, 95 \%$ confidence interval; $\mathrm{BMI}$, body mass index; $\mathrm{BMD}$, bone mineral density.

routinely in most hospitals due to patients' unwillingness to undergo additional examinations. Zou et al introduced the HU system as a practical measurement tool utilizing lumbar $\mathrm{CT}$ to evaluate bone mineral density in patients with DDD without any extra cost. ${ }^{16}$ Many studies have also demonstrated a strong positive correlation between the $\mathrm{HU}$ value and BMD, with excellent inter-and intrarater reliability. ${ }^{15,35}$ Meanwhile, the TEPS system, introduced by Rajasekaran et al, was also widely accepted by clinicians for its higher correlation with the incidence of DDD. ${ }^{10,36}$

We present the first clinical evidence of the association between osteoporosis and endplate damage with clinical and radiological data employing universally accepted methods. Our study gathered cross-sectional data and rigorously analyzed the HU values and TEPS applying univariate and multivariate regression. We observed a significant negative correlation between these two factors in our patients with DDD, indicating a relationship between osteoporosis and endplate damage. Our clinical findings corroborate the results of the animal model studies mentioned above.

Considering surgical complications related to osteoporosis, such as delayed lumbar fusion, loss of reduction, cage subsidence, and screw loosening, ${ }^{8,37,38}$ it is of great significance for surgeons to recognize and manage patients with osteoporosis appropriately before performing surgeries. Previous studies have found that some anti-osteoporosis drugs, such as alendronate sodium, zoledronate, and denosumab, could slow lumbar disc degeneration. ${ }^{39}$ Our clinical studies also support the theory that osteoporosis patients are more likely to experience endplate damage and even DDD associated with lower BMD. This serves as an indication for anti-osteoporosis treatment in the perioperative period in patients with DDD. Furthermore, our study proposed that the metabolic relevance of endplate damage and osteoporosis might serve as a promising quantifiable benchmark for future pharmacological randomized controlled studies. ${ }^{40}$

We also attempted to explain the underlying mechanism that accounts for the correlation between osteoporosis and endplate damage. Osteoclasts are one of the major cells that regulate bone mass, and chondrocytes are known to be important components of cartilaginous endplates. Park et al demonstrated that blocking osteoclastassociated receptors reduced articular cartilage destruction via regulation of chondrocyte apoptosis. ${ }^{41}$ Xiao et al observed a significant increase in osteoclasts in the endplate based on an ovariectomy mouse model. ${ }^{42}$ Thus, we hypothesized that a close connection between osteoclasts and endplate chondrocytes exists due to the high structural and functional similarity of the cartilaginous endplate and the articular cartilage. Further experiments and longitudinal studies are required to confirm this hypothesis.

There were some limitations to this study. The factors investigated in this study were purely radiological and subjectively measured. Although they were gathered and calculated by experienced doctors, measurement bias remains a possibility. Furthermore, direct comparisons between DXA and CT HU values were not performed because DXA measurements were not available for the majority of patients with DDD, as outlined above. Furthermore, the cross-sectional nature of this study limited our ability to define the causality 
between the two abnormalities. However, it was possible to confirm the mutual interaction between osteoporosis and endplate damage.

\section{Conclusion}

Our cross-sectional study on human subjects demonstrates the first clinical evidence of a significant positive correlation between osteoporosis and endplate damage. More careful consideration by clinicians, a detailed diagnosis, and comprehensive treatment plan are recommended when treating patients with DDD or osteoporosis-related complications.

\section{Ethics Approval and Informed Consent}

This study's protocol was approved by the institutional ethical committee of Zhongshan Hospital (B2021-388) and was performed according to the principles of the Declaration of Helsinki of 1975 . Written and verbal informed consent was obtained from all participants prior to study inclusion and all the data was anonymized and confidential.

\section{Acknowledgments}

This study was supported by the Shanghai Sailing Program from Shanghai Science and Technology Committee (No. 18YF1404400).

\section{Disclosure}

The authors declare that they have no conflicts of interest.

\section{References}

1. Chen P, Li Z, Hu Y. Prevalence of osteoporosis in China: a meta-analysis and systematic review. BMC Public Health. 2016;16 (1):1039. doi:10.1186/s12889-016-3712-7

2. Curry SJ, Krist AH, Owens DK, et al. Screening for osteoporosis to prevent fractures: US Preventive Services Task Force recommendation statement. JAMA. 2018;319(24):2521-2531. doi:10.1001/jama.20 18.7498

3. Cheung KM, Karppinen J, Chan D, et al. Prevalence and pattern of lumbar magnetic resonance imaging changes in a population study of one thousand forty-three individuals. Spine (Phila Pa 1976). 2009;34 (9):934-940. doi:10.1097/BRS.0b013e3181a01b3f

4. Kalichman L, Kim DH, Li L, Guermazi A, Hunter DJ. Computed tomography-evaluated features of spinal degeneration: prevalence, intercorrelation, and association with self-reported low back pain. Spine J. 2010;10(3):200-208. doi:10.1016/j.spinee.2009.10.018

5. Samartzis D, Karppinen J, Chan D, Luk KD, Cheung KM. The association of lumbar intervertebral disc degeneration on magnetic resonance imaging with body mass index in overweight and obese adults: a population-based study. Arthritis Rheum. 2012;64 (5):1488-1496. doi:10.1002/art.33462
6. Chan WC, Sze KL, Samartzis D, Leung VY, Chan D. Structure and biology of the intervertebral disk in health and disease. Orthop Clin North Am. 2011;42(4):447-464, vii. doi:10.1016/j.ocl.2011.07.012

7. Rade M, Maatta JH, Freidin MB, Airaksinen O, Karppinen J, Williams FMK. Vertebral endplate defect as initiating factor in intervertebral disc degeneration: strong association between endplate defect and disc degeneration in the general population. Spine (Phila $P a$ 1976). 2018;43(6):412-419. doi:10.1097/BRS.00000000 00002352

8. Mi J, Li K, Zhao X, Zhao CQ, Li H, Zhao J. Vertebral body hounsfield units are associated with cage subsidence after transforaminal lumbar interbody fusion with unilateral pedicle screw fixation. Clin Spine Surg. 2017;30(8):E1130-e1136. doi:10.1097/BSD.000000000 0000490

9. Ullrich BW, Schenk P, Spiegl UJ, Mendel T, Hofmann GO. Hounsfield units as predictor for cage subsidence and loss of reduction: following posterior-anterior stabilization in thoracolumbar spine fractures. Eur Spine J. 2018;27(12):3034-3042. doi:10.1007/s00586018-5792-9

10. Munir S, Freidin MB, Rade M, Määttä J, Livshits G, Williams FMK. Endplate defect is heritable, associated with low back pain and triggers intervertebral disc degeneration: a longitudinal study from twinsUK. Spine (Phila Pa 1976). 2018;43(21):1496-1501. doi:10.1097/BRS.0000000000002721

11. Ding Y, Jiang J, Zhou J, et al. The effects of osteoporosis and disc degeneration on vertebral cartilage endplate lesions in rats. Eur Spine J. 2014;23(9):1848-1855. doi:10.1007/s00586-014-3324-9

12. Jain RK, Vokes T. Dual-energy X-ray absorptiometry. $J$ Clin Densitom. 2017;20(3):291-303. doi:10.1016/j.jocd.2017.06.014

13. Donescu OS, Battié MC, Videman T. The influence of magnetic resonance imaging findings of degenerative disease on dual-energy X-ray absorptiometry measurements in middle-aged men. Acta Radiol. 2007;48(2):193-199. doi:10.1080/02841850601129015

14. Pan J, Lu X, Yang G, Han Y, Tong X, Wang Y. Lumbar disc degeneration was not related to spine and hip bone mineral densities in Chinese: facet joint osteoarthritis may confound the association. Arch Osteoporos. 2017;12(1):20. doi:10.1007/s11657-017-0315-6

15. Schreiber JJ, Anderson PA, Rosas HG, Buchholz AL, Au AG. Hounsfield units for assessing bone mineral density and strength: a tool for osteoporosis management. J Bone Joint Surg Am. 2011;93(11):1057-1063. doi:10.2106/JBJS.J.00160

16. Zou D, Li W, Deng C, Du G, Xu N. The use of CT Hounsfield unit values to identify the undiagnosed spinal osteoporosis in patients with lumbar degenerative diseases. Eur Spine J. 2019;28 (8):1758-1766. doi:10.1007/s00586-018-5776-9

17. Rajasekaran S, Venkatadass K, Naresh Babu J, Ganesh K, Shetty AP. Pharmacological enhancement of disc diffusion and differentiation of healthy, ageing and degenerated discs: results from in-vivo serial post-contrast MRI studies in 365 human lumbar discs. Eur Spine $J$. 2008;17(5):626-643. doi:10.1007/s00586-008-0645-6

18. Kaiser MG, Eck JC, Groff MW, et al. Guideline update for the performance of fusion procedures for degenerative disease of the lumbar spine. Part 1: introduction and methodology. J Neurosurg Spine. 2014;21(1):2-6. doi:10.3171/2014.4.SPINE14257

19. Zaidi Q, Danisa OA, Cheng W. Measurement techniques and utility of hounsfield unit values for assessment of bone quality prior to spinal instrumentation. Spine. 2019;44(4):E239-E244. doi:10.1097/ BRS.000000000002813

20. Decker RS, Koyama E, Pacifici M. Articular cartilage: structural and developmental intricacies and questions. Curr Osteoporos Rep. 2015;13(6):407-414. doi:10.1007/s11914-015-0290-z

21. Horner HA, Urban JP. 2001 volvo award winner in basic science studies: effect of nutrient supply on the viability of cells from the nucleus pulposus of the intervertebral disc. Spine (Phila Pa 1976). 2001;26(23):2543-2549. doi:10.1097/00007632-200112010-00006 
22. Calvo E, Castañeda S, Largo R, Fernández-Valle ME, RodríguezSalvanés F, Herrero-Beaumont G. Osteoporosis increases the severity of cartilage damage in an experimental model of osteoarthritis in rabbits. Osteoarthritis Cartilage. 2007;15(1):69-77. doi:10.1016/j. joca.2006.06.006

23. Bertuglia A, Lacourt M, Girard C, Beauchamp G, Richard H, Laverty S. Osteoclasts are recruited to the subchondral bone in naturally occurring post-traumatic equine carpal osteoarthritis and may contribute to cartilage degradation. Osteoarthritis Cartilage. 2016;24(3):555-566. doi:10.1016/j.joca.2015.10.008

24. Çarlı AB, Akarsu S, Tekin L, Sağlam M, Kıralp MZ, Özçakar L. Ultrasonographic assessment of the femoral cartilage in osteoarthritis patients with and without osteoporosis. Aging Clin Exp Res. 2014;26 (4):411-415. doi:10.1007/s40520-013-0188-5

25. Zou D, Jiang S, Zhou S, et al. Prevalence of osteoporosis in patients undergoing lumbar fusion for lumbar degenerative diseases: a combination of DXA and hounsfield units. Spine (Phila Pa 1976). 2020;45(7):E406-e410. doi:10.1097/BRS.0000000000003284

26. Fehlings D, Switzer L, Agarwal P, et al. Informing evidence-based clinical practice guidelines for children with cerebral palsy at risk of osteoporosis: a systematic review. Dev Med Child Neurol. 2012;54 (2):106-116. doi:10.1111/j.1469-8749.2011.04091.x

27. Grams AE, Rehwald R, Bartsch A, et al. Correlation between degenerative spine disease and bone marrow density: a retrospective investigation. BMC Med Imaging. 2016;16:17. doi:10.1186/s12880016-0123-2

28. Harada A, Okuizumi H, Miyagi N, Genda E. Correlation between bone mineral density and intervertebral disc degeneration. Spine (Phila Pa 1976). 1998;23(8):857-861; discussion 862. doi:10.1097/ 00007632-199804150-00003

29. Salo S, Leinonen V, Rikkonen T, et al. Association between bone mineral density and lumbar disc degeneration. Maturitas. 2014;79 (4):449-455. doi:10.1016/j.maturitas.2014.09.003

30. Wang YX, Griffith JF, Ma HT, et al. Relationship between gender, bone mineral density, and disc degeneration in the lumbar spine: a study in elderly subjects using an eight-level MRI-based disc degeneration grading system. Osteoporos Int. 2011;22(1):91-96. doi:10.1007/s00198-010-1200-y

31. Wang YX, Kwok AW, Griffith JF, et al. Relationship between hip bone mineral density and lumbar disc degeneration: a study in elderly subjects using an eight-level MRI-based disc degeneration grading system. J Magn Reson Imaging. 2011;33(4):916-920. doi:10.1002/ jmri.22518

32. Zhou S, Zhu L, You T, et al. In vivo quantification of bone mineral density of lumbar vertebrae using fast $\mathrm{kVp}$ switching dual-energy CT: correlation with quantitative computed tomography. Quant Imaging Med Surg. 2021;11(1):341-350. doi:10.21037/qims-20-367
33. Woisetschlager M, Hagg M, Spangeus A. Computed tomography-based opportunistic osteoporosis assessment: a comparison of two software applications for lumbar vertebral volumetric bone mineral density measurements. Quant Imaging Med Surg. 2021;11(4):1333-1342. doi:10.21037/qims-20-1013

34. Okano I, Salzmann SN, Jones C, et al. The impact of degenerative disc disease on regional volumetric bone mineral density (vBMD) measured by quantitative computed tomography. Spine J. 2020;20 (2):181-190. doi:10.1016/j.spinee.2019.02.017

35. Hendrickson NR, Pickhardt PJ, Del Rio AM, Rosas HG, Anderson PA. Bone mineral density T-scores derived from CT attenuation numbers (hounsfield units): clinical utility and correlation with dual-energy X-ray absorptiometry. Iowa Orthop J. 2018;43:25-31.

36. Pradip IA, Dilip Chand Raja S, Rajasekaran S, et al. Presence of preoperative Modic changes and severity of endplate damage score are independent risk factors for developing postoperative surgical site infection: a retrospective case-control study of 1124 patients. Eur Spine J. 2020. doi:10.1007/s00586-020-06581-7

37. Bokov A, Bulkin A, Aleynik A, Kutlaeva M, Mlyavykh S. Pedicle screws loosening in patients with degenerative diseases of the lumbar spine: potential risk factors and relative contribution. Global Spine J. 2019;9(1):55-61. doi:10.1177/2192568218772302

38. Schreiber JJ, Hughes AP, Taher F, Girardi FP. An association can be found between hounsfield units and success of lumbar spine fusion. HSS J. 2014;10(1):25-29. doi:10.1007/s11420-013-9367-3

39. Cai G, Laslett LL, Aitken D, et al. Effect of zoledronic acid and denosumab in patients with low back pain and modic change: a proof-of-principle trial. J Bone Miner Res. 2018;33(5):773-782. doi:10.1002/jbmr.3376

40. Maugeri D, Panebianco P, Russo MS, et al. Ipriflavone-treatment of senile osteoporosis: results of a multicenter, double-blind clinical trial of 2 years. Arch Gerontol Geriatr. 1994;19(3):253-263. doi:10.1016/ 0167-4943(94)00571-0

41. Park DR, Kim J, Kim GM, et al. Osteoclast-associated receptor blockade prevents articular cartilage destruction via chondrocyte apoptosis regulation. Nat Commun. 2020;11(1):4343. doi:10.1038/ s41467-020-18208-y

42. Xiao ZF, He JB, Su GY, et al. Osteoporosis of the vertebra and osteochondral remodeling of the endplate causes intervertebral disc degeneration in ovariectomized mice. Arthritis Res Ther. 2018;20 (1):207. doi:10.1186/s13075-018-1701-1
Clinical Interventions in Aging

\section{Publish your work in this journal}

Clinical Interventions in Aging is an international, peer-reviewed journal focusing on evidence-based reports on the value or lack thereof of treatments intended to prevent or delay the onset of maladaptive correlates of aging in human beings. This journal is indexed on PubMed Central, MedLine, CAS, Scopus and the Elsevier
Bibliographic databases. The manuscript management system is completely online and includes a very quick and fair peer-review system, which is all easy to use. Visit http://www.dovepress.com/ testimonials.php to read real quotes from published authors. 